\title{
Serum CCAT2 as a biomarker for adjuvant diagnosis and prognostic prediction of cervical cancer
}

Xiaoli $\mathrm{CaO}^{1 \dagger}{ }^{\dagger}$, Juan $\mathrm{Yao}^{2 \dagger}$, Meiqun Jia ${ }^{2}$, Xianjuan Shen ${ }^{3}$, Jinye Zhang ${ }^{1 *}$ and Shaoqing $\mathrm{Ju}^{3^{*}}$ (1)

\begin{abstract}
Growing evidence indicates that IncRNA colon cancer-associated transcript 2 (CCAT2) is associated with cancers. However, the clinical value of CCAT2 in cervical cancer (CC) remains unclear. In this study, serum CCAT2 level was detected by real-time quantitative PCR (RT-qPCR). Carbohydrate antigen 125 (CA125) and squamous-cell carcinoma antigen (SCC) were detected by electrochemiluminescence. A receiver operating characteristic (ROC) curve was utilized to estimate the diagnostic efficiency of CCAT2. Kaplan-Meier survival analysis and univariable and multivariable analyses were performed to assess the prognostic value of CCAT2. The relative expression level of CCAT2 in primary CC patients was significantly higher than that in cervical intraepithelial neoplasias (CIN) patients and healthy controls (both $P<0.001$ ). CCAT2 relative expression was positively correlated with tumor Federation of Gynecology and Obstetrics (FIGO) stage, SCC-Ag and lymph node metastasis (LNM) (all $P<0.05)$. CCAT2 expression in recurrent/ metastatic CC was significantly higher compared with primary CC $(P<0.0001)$ or operated CC $(P<0.0001)$ and during follow-up, CCAT2 expression was increased before surgery and decreased significantly after surgery $(P<0.0001)$. Furthermore, the overall survival rate of CC patients with high CCAT2 expression group markedly decreased as compared with that of low CCAT2 expression group $(P=0.026)$. Univariate analyses indicated that CCAT2 was a poor prognostic factor associated with overall survival (OS). Our study indicates that CCAT2 may be valuable in complementary diagnosis and monitoring of progression and prognosis of CC patients. Combined detection of CCAT2, CA125 and SCC can greatly improve the diagnostic efficiency of primary CC.
\end{abstract}

Keywords: Cervical carcinoma, IncRNA CCAT2, Real-time quantitative PCR

\section{Introduction}

Cervical cancer (CC) ranks the second in female malignant tumors, and the second cause of cancer-related death in cancer patients aged 20-39years. In addition, the incidence of CC tends to increase gradually each year

\footnotetext{
*Correspondence: jykzjy@126.com; jsq814@hotmail.com

tXiaoli Cao and Juan Yao contributed equally to this work.

${ }^{1}$ Department of Laboratory Medicine, Affiliated Tumor Hospital

of Nantong University, \#48 West Qingnian Road, Nantong 226019, Jiangsu Province, China

${ }^{3}$ Laboratory Medicine Center, Affiliated Hospital of Nantong University, \#20 Xisi Road, Nantong 226001, Jiangsu Province, China

Full list of author information is available at the end of the article
}

[1]. Early surgical intervention, radiotherapy and chemotherapy can offer a high survival rate in CC patients. However, many CC patients have already been in the middle or late state and lost the chance of surgery at the time of diagnosis. Radiochemotherapy for such patients is often ineffective and their survival and prognosis are usually poor. With technical advances in recent years, tumor markers have gradually been used for early screening of malignant tumors due to simplicity, microinvasiveness and quickness, and play a significant role in early diagnosis, therapeutic assessment and prognostic prediction of cancer patients.

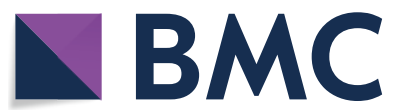

(c) The Author(s) 2022. Open Access This article is licensed under a Creative Commons Attribution 4.0 International License, which permits use, sharing, adaptation, distribution and reproduction in any medium or format, as long as you give appropriate credit to the original author(s) and the source, provide a link to the Creative Commons licence, and indicate if changes were made. The images or other third party material in this article are included in the article's Creative Commons licence, unless indicated otherwise in a credit line to the material. If material is not included in the article's Creative Commons licence and your intended use is not permitted by statutory regulation or exceeds the permitted use, you will need to obtain permission directly from the copyright holder. To view a copy of this licence, visit http://creativecommons.org/licenses/by/4.0/. The Creative Commons Public Domain Dedication waiver (http://creativeco mmons.org/publicdomain/zero/1.0/) applies to the data made available in this article, unless otherwise stated in a credit line to the data. 
Carcinoembryonic antigen (CEA), carbohydrate antigen 125 (CA125) and squamous cell carcinoma antigen (SCC) are three main tumor markers for early screening, therapeutic monitoring and prognostic assessment of $\mathrm{CC}$ at present. But as they are not sensitive and specific as expected, more reliable, sensitive and specific serum markers are required.

LncRNA colon cancer associated transcript 2 (CCAT2) is highly expressed in colon cancer and can promote tumor growth, metastasis and chromosomal instability. He et al. [2] found CCAT2 promoted prostate cancer cell proliferation and invasion by regulating the Wnt/ $\beta$-catenin signaling pathway. Sun et al. [3] reported that low expression of miR-424 in normal human astrocytes (NHA) was accompanied with a high expression of CCAT2 and vascular endothelial growth factor-A (VEGFA). Xu et al [4] discovered that CCAT2 promoted the development and progression of triple-negative breast cancer (TNBC) by up-regulating the expression of OCT4-PG1 and activating Notch signaling. Roxana et al. [5] first analyzed the expression of CCAT2 in normal breast tissue and breast cancer tissue by RT-qPCR and observed that breast cancer patients with high CCAT2 expression could not benefit from cyclophosphamide (CTX) +5-fluorouracil (5-FU) + methotrexate (CMF) adjuvant chemotherapy, and these patients often had a shorter survival duration. CCAT2 expression in liver cancer tissue was significantly higher than that in normal control. High CCAT2 expression is an independent risk factor of predicting shorter survival of hepatocellular carcinoma (HCC) patients [6].

We hypothesize that there is a correlation between CCAT2 and CC. In the present study, we explored CCAT2 expression in CC and evaluated the value as a serum biomarker for clinical adjuvant diagnosis and prognostic prediction of $\mathrm{CC}$.

\section{Materials and methods Sample collection}

Serum samples were collected from 180 primary CC patients aged $28-76$ years with a median of $52(45.0,58.0)$ years, 165 postoperative CC patients aged 28-76years with a median of 51 (46.0, 58.0) years, 44 inoperable recurrent and metastatic CC patients aged 31-70years with a median of $54(49.0,60.0)$ years, $80 \mathrm{CIN}$ patients aged $23-74$ years with a median of $47(40.0,51.5)$ years, and 100 healthy individuals aged 24-69years with a median of 48 (40.0, 56.0) years as control. In addition, serum samples were collected consecutively from 30 of the 180 primary CC patients who underwent operation during the follow-up periods of 1-180 days. The primary CC patients and CIN patients were selected from the patients who were clinic pathologically confirmed as having $\mathrm{CC}$ and received treatment in the department of gynecology of Nantong Tumor Hospital between January 2015 and December 2018. The healthy controls were selected from individuals who underwent physical examination in the PE Center of the same hospital during the same period. Tumor staging and typing were according to the American Joint Committee on Cancer (AJCC) guidelines [7]. This research protocol was approved by the ethics committee of the said hospital (LW2020002), and all samples were anonymous. Informed consent was obtained from all patients and controls. The blood samples were from the remaining samples that used for routine examination. Serum samples $(5 \mathrm{ml}$ each) were centrifuged at $1000 \mathrm{r} / \mathrm{min}$ for $10 \mathrm{~min}$ to collect the sera, which were restored at $80^{\circ} \mathrm{C}$ for use.

\section{RNA extraction and CDNA synthesis}

Serum total RNA was extracted using the serum RNA extraction kit (Beijing Patek Biotechnology Co., Ltd., Beijing, China). RNA optical density (OD) was measured by ultraviolet spectrophotometry to calculate the $\mathrm{OD}_{260} / \mathrm{OD}_{280}$ ratio of the RNA samples, knowing that the ratio between 1.8 and 2.0 indicates good purity of the RNA extracted. The extracted RNA was reverse transcribed into cDNA by using Revert Aid First Strand cDNA Synthesis Kit (Thermo, USA). The reaction system is as follows: $300 \mathrm{ng}$ RNA, $4 \mu \mathrm{l} 5 \times$ reaction buffer, $2 \mu \mathrm{l}$ $(10 \mathrm{nM})$ deoxynucleoside triphosphate (dNTP), $1 \mu \mathrm{l}$ oligonucleotide as the primer $(\mathrm{dT}), 1 \mu \mathrm{l}(200 \mathrm{U} / \mu \mathrm{l})$ reverse transcriptase ribonuclease inhibitor, and addition of enzyme-free $\mathrm{H}_{2} \mathrm{O}$ to $20 \mu \mathrm{l}$. They were mixed thoroughly, centrifuged and reverse transcribed at $42^{\circ} \mathrm{C}$ for $60 \mathrm{~min}$ and $70^{\circ} \mathrm{C}$ for $5 \mathrm{~min}$.

\section{RT-qPCR assay}

RT-qPCR assay was performed with Light Cycler 480 RT-QPCR using the following reaction system: $10 \mu \mathrm{l}$ SYBR Green I mix, $3 \mu \mathrm{l}$ cDNA, $1 \mu \mathrm{l}$ up-stream primer, $1 \mu \mathrm{l}$ down-stream primer, and $5 \mu$ enzyme-free $\mathrm{H}_{2} \mathrm{O}$ to a total volume of $20 \mu \mathrm{l}$ under the reaction condition of $95^{\circ} \mathrm{C}$ for $5 \mathrm{~min}$, one cycle; $95^{\circ} \mathrm{C}, 15 \mathrm{~s} ; 61^{\circ} \mathrm{C}, 30 \mathrm{~s} ; 72^{\circ} \mathrm{C}, 30 \mathrm{~s}$, totaling 45 cycles. Three wells were set for each sample and the mean value was used for analysis. IncRNA relative expression $(R Q)=2^{-\triangle \triangle C T}$ was used to indicate the relative expression of CCAT2, and $\triangle \triangle$ cycle threshold $(\mathrm{Ct})=$ study group $\left(\mathrm{CT}_{\mathrm{CCAT2} 2}-\mathrm{CT}_{\mathrm{GAPDH}}\right)$--control

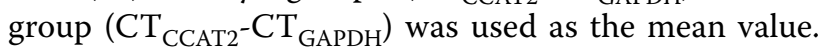
The primer sequences are as follows: CCAT2 up-stream primer: 5'-CCCTGGTCAAATTGCTAAACCT-3', down-stream primer: 5'-TTATTCGTCCCTCTGTTT TATGGAT-3'; GAPDH up-stream primer: 5'-TGA TGACATCAAGAAGGTGGTGAAG-3', down-stream primer: 5'-TCCTTGGAGGCCCAGTGGGCCAT-3'. 


\section{Methodological evaluation}

CA125 and SCC were detected by electrochemiluminescence using the E601 electrochemiluminescence instrument (Roche, Germany) and Maglumi automatic chemiluminescence instrument (New industry Biomedical Engineering Co., Ltd., Shenzhen, China) respectively.

\section{Follow-up}

We collected information on 5-year survivors from 154 of the 180 primary CC patients. Follow-up with all CC patients occurred by telephone once every 3 months in the first 2 years and every 6 months after that.

\section{Statistical analysis}

Statistical analysis and ROC curve mapping were performed by SPSS20.0. Pair-wise comparison of CCAT2 relative expression between primary $\mathrm{CC}$ patients, CIN patients and healthy controls was performed by MannWhitney U test. The correlation between CCAT2 relative expression and clinicopathological features was analyzed by $\chi^{2}$ test. OS was analyzed by Kaplan-Meier analysis. Univariate and multivariate analyses were evaluated with Cox proportional hazards models. Mapping was performed by GraphPad Prism 5. Statistically significant difference was set as $P<0.05$.

\section{Results}

Serum relative expression of CCAT2 in primary patients, CIN patients and healthy controls

The median value of serum CCAT2 relative expression in the 100 healthy controls was $0.727(0.544,0.990)$ and $0.795(0.591,0.979)$ in the $80 \mathrm{CIN}$ patients. There was no significant difference in serum CCAT2 relative expression between CIN patients and healthy controls $(P>0.05)$. The median value of serum CCAT2 relative expression in 180 primary CC patients was 1.616 (1.068, $2.219)$, which was significantly higher than that in CIN patients and healthy controls (both $P<0.001$ ) (Fig. 1).

\section{Correlation between serum CCAT2 relative expression} and clinicopathological features of primary CC patients Correlation analysis of serum CCAT2 relative expression with age, menopause, tumor size, FIGO stage, pathological type, SCC-Ag and the presence or absence of LNM in 180 primary CC patients showed that serum CCAT2 relative expression was positively correlated with tumor FIGO stage, SCC-Ag and LNM (all $P<0.05$ ) (Table 1 ).

\section{Application of CCAT2, CA125 and SCC detection} to diagnosis of primary CC

Pairwise comparison demonstrated that the serum concentration of CA125 in 180 primary CC patients was $16.14(10.55,23.08) \mathrm{U} / \mathrm{ml}$ vs. $11.50(7.53,16.50) \mathrm{U} / \mathrm{ml}$

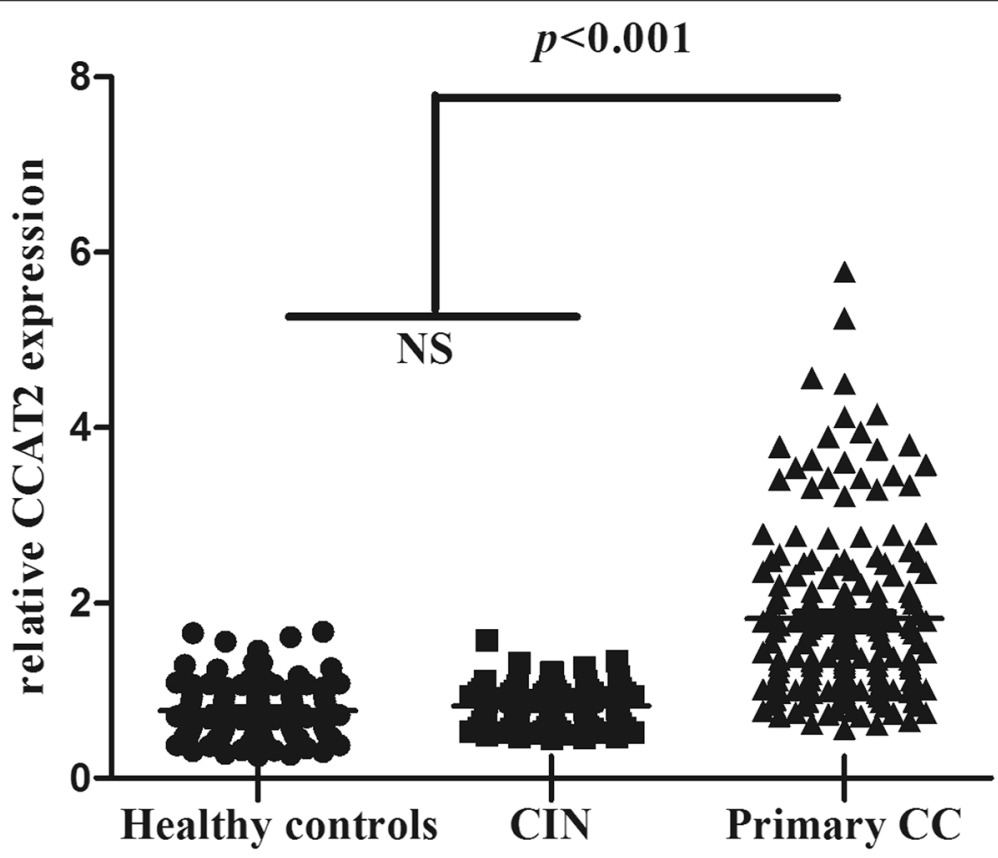

Fig. 1 A scatter diagram of serum CCAT2 in healthy controls, CIN patients and primary CC patients. CCAT2 was detected by RT-qPCR. Pair-wise comparison of the relative expression of CCAT2 between primary CC patients, CIN patients and healthy controls was performed by Mann-Whitney $U$ test, and $P<0.05$ was considered statistically significant. NS: no significance 
Table 1 Correlation between serum CCAT2 relative expression and clinicopathological features of CC patients

\begin{tabular}{|c|c|c|c|}
\hline Clinicopathologicfeatures & $n=$ & $\begin{array}{l}\text { CCAT2 relative expression } \\
\text { [median (upper and lower quartile)] }\end{array}$ & Pvalue \\
\hline Total $(n)$ & 180 & & \\
\hline Age (yr) & & & 0.222 \\
\hline$\leq 50$ & 79 & $1.434(1.050,2.343)$ & \\
\hline$>50$ & 101 & $1.679(1.079,2.131)$ & \\
\hline Menopause & & & 0.460 \\
\hline yes & 94 & $1.586(1.043,2.282)$ & \\
\hline no & 86 & $1.675(1.110,2.219)$ & \\
\hline Tumor size & & & 0.101 \\
\hline$\leq 4 \mathrm{~cm}$ & 90 & $1.357(1.021,2.129)$ & \\
\hline$>4 \mathrm{~cm}$ & 90 & $1.749(1.181,2.343)$ & \\
\hline FIGO stage & & & $0.038^{*}$ \\
\hline$|A 1-| b \mid$ & 63 & $1.283(1.007,2.056)$ & \\
\hline |b2-1Ia2 & 112 & $1.722(1.189,2.298)$ & \\
\hline$\geq 11 \mathrm{~b}$ & 5 & $3.411(1.052,3.822)$ & \\
\hline Pathological type & & & 0.263 \\
\hline aquamous $\mathrm{Ca}$ & 152 & $1.675(1.110,2.131)$ & \\
\hline adenal Ca & 22 & $1.503(1.021,3.541)$ & \\
\hline adenosqua. Ca & 6 & $1.153(0.924,1.357)$ & \\
\hline SCC-Ag (ng/ml) & & & $0.001^{*}$ \\
\hline negative & 98 & $1.352(1.014,1.866)$ & \\
\hline positive & 82 & $1.886(1.266,2.453)$ & \\
\hline LNM & & & $0.046^{*}$ \\
\hline negative & 150 & $1.517(1.047,2.129)$ & \\
\hline positive & 30 & $1.904(1.257,2.596)$ & \\
\hline
\end{tabular}

${ }^{*} P<0.05$; FIGO: International Federation of Gynecology and Obstetrics; CC Cervical cancer CCAT2 Colon cancer-associated transcript 2, LNM Lymph node metastasis

in 100 healthy controls, showing a significant difference between the two groups $(P<0.05)$. The serum concentration of SCC and CCAT2 in primary CC patients was 2.10 $(1.20,6.60) \mathrm{ng} / \mathrm{ml}$ vs. $0.90(0.60,1.30) \mathrm{ng} / \mathrm{ml}$ in healthy controls and $1.616(1.068,2.219)$ vs. $0.727(0.544,0.990)$ respectively, showing significant differences between the two groups $(P<0.001)$ (Table 2$)$.

Serum CCAT2 relative expression was not significantly correlated with the CA125 concentration in 180 primary CC patients $\left(P=0.926, r^{2}<0.0001\right)$ but significantly correlated with the SCC concentration $(P<0.0001$, $\left.r^{2}=0.120\right)$ (Figs. 2, 3).
Evaluation of the diagnostic value of serum CCAT2, CA125 or SCC for primary CC

When the cutoff value of CCAT2 for diagnosing CC was 1.102 (73.33\%sensitivity and $87.00 \%$ specificity), the AUC (area under the curve) for CCAT2 was 0.897 (95\%CI: $0.862-0.933)$. By that analogy, the cutoff value used for CA125 was $9.69 \mathrm{U} / \mathrm{ml}(81.67 \%$ sensitivity and $46.00 \%$ specificity). The AUC for CA125 was 0.678 (95\%CI: $0.614-0.743)$. The cutoff value used for SCC was $1.55 \mathrm{ng} /$ $\mathrm{ml}(69.44 \%$ sensitivity and $90.00 \%$ specificity). The area under the curve for SCC was 0.815 (95\%CI: 0.767-0.863) (Fig. 4).

Table 2 The median and cutoff values of CCAT2, CA125 and SCC in primary CC patients and healthy controls

\begin{tabular}{llllll}
\hline Parameters & Primary CC & Healthy controls & Cutoff & AUC & $\boldsymbol{p}$ \\
\hline CCAT2 & $1.616(1.068,2.219)$ & $0.727(0.544,0.990)$ & 1.102 & 0.897 & $<0.001$ \\
CA125 $(\mathrm{U} / \mathrm{ml})$ & $16.14(10.55,23.08)$ & $11.50(7.53,16.50)$ & 9.69 & 0.678 & $<0.05$ \\
SCC $(\mathrm{ng} / \mathrm{ml})$ & $2.10(1.20,6.60)$ & $0.90(0.60,1.30)$ & 1.55 & 0.815 & $<0.001$ \\
\hline
\end{tabular}




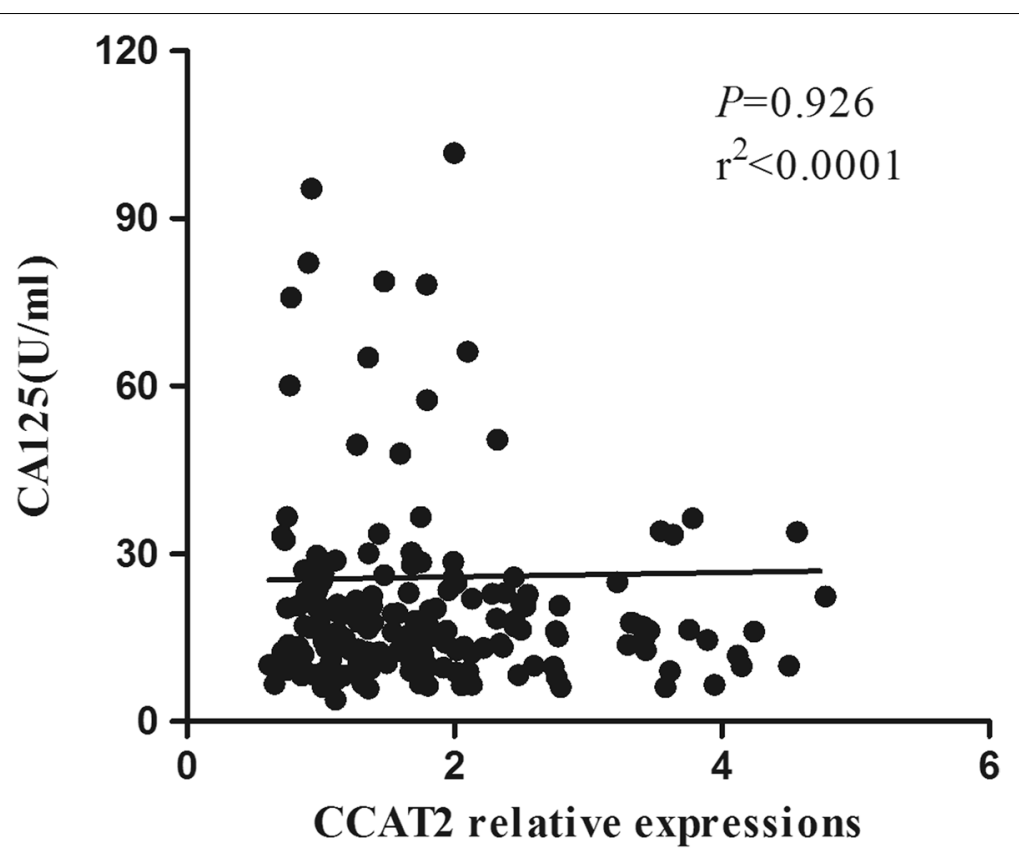

Fig. 2 A scatter diagram of CCAT2 and CA125 correlation in primary CC patients, $P=0.926, r^{2}<0.0001$

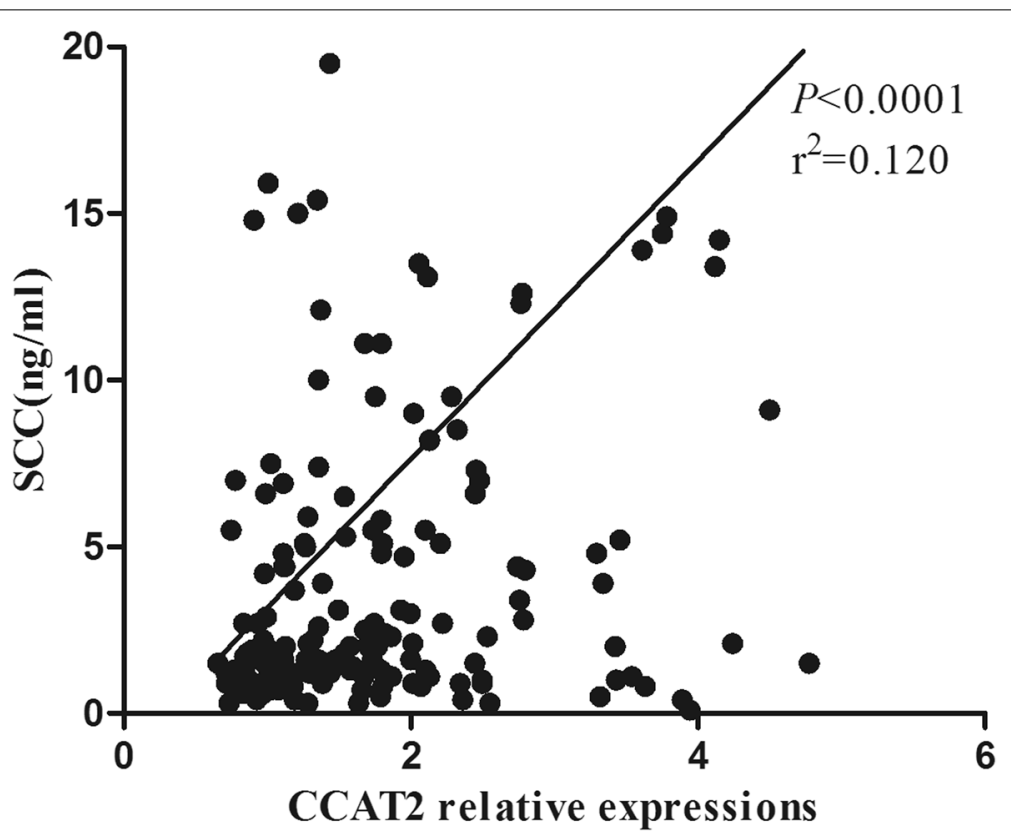

Fig. 3 A scatter diagram of CCAT2 and SCC correlation in primary CC patients, $P<0.0001, r^{2}=0.120$

Next, a combined analysis of CCAT2, CA125 and SCC was performed using a tandem model of the three markers. The sensitivity, specificity, accuracy, positive prediction and negative prediction of this panel in differentiating the healthy controls were 94.44, 96.00, $95.00,97.70$ and $90.57 \%$ respectively, which greatly improved the diagnostic efficiency in diagnosing CC (Table 3). 


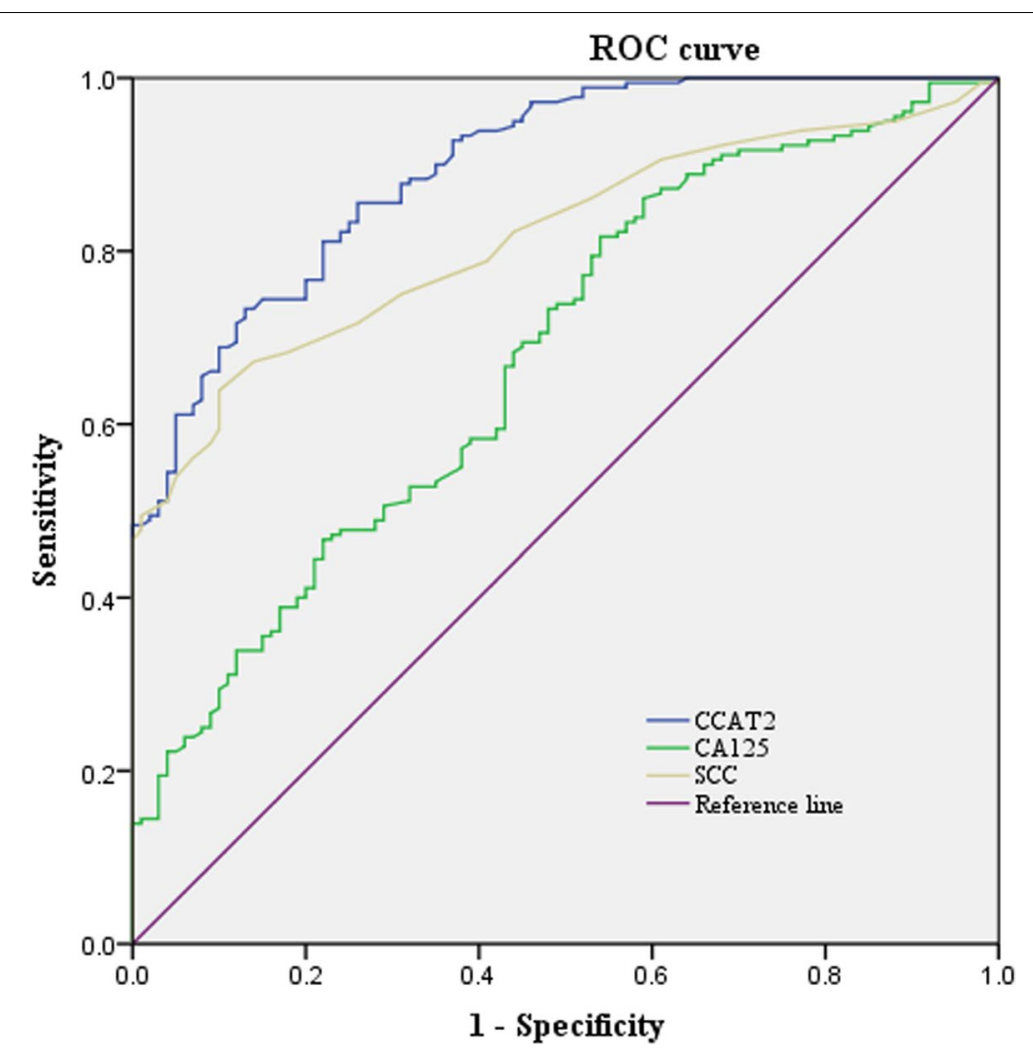

Fig. 4 RCO differentiation between primary CC patients and healthy controls. The AUC value of CCAT2, CA125 and SCC was 0.897 (95\%Cl: $0.862-$ 0.933), 0.678 (95\%Cl:0.614-0.743) and 0.815 (95\%Cl:0.767-0.863), respectively

Table 3 The value of CCAT2, CA125 and SCC in diagnosing primary CC

\begin{tabular}{llllll}
\hline Molecular marker & Sensitivity & Specificity & Accuracy & Positive prediction & $\begin{array}{l}\text { Negative } \\
\text { prediction } \\
\text { (\%) }\end{array}$ \\
\hline CCAT2 & $\mathbf{( \% )}$ & $\mathbf{( \% )}$ & $\mathbf{( \% )}$ & $\mathbf{( \% )}$ & 64.44 \\
& 73.33 & 87.00 & 78.21 & 91.03 & $(87 / 135)$ \\
CA125 & $(132 / 180)$ & $(87 / 100)$ & $(219 / 280)$ & $(132 / 145)$ & 58.23 \\
& 81.67 & 46.00 & 68.93 & 73.13 & $(46 / 79)$ \\
SCC & $(147 / 180)$ & $(46 / 100)$ & $(193 / 280)$ & $(147 / 201)$ & 62.07 \\
& 69.44 & 90.00 & 76.79 & 92.59 & $(90 / 145)$ \\
CCAT2+CA125 & $(125 / 180)$ & $(90 / 100)$ & $(215 / 280)$ & $(125 / 135)$ & 85.58 \\
& 91.67 & 89.00 & 90.71 & 93.75 & $(89 / 104)$ \\
CCAT2+SCC & $(165 / 180)$ & $(89 / 100)$ & $(254 / 280)$ & $(165 / 176)$ & 75.00 \\
& 82.22 & 96.00 & 87.14 & 97.37 & $(96 / 128)$ \\
Three combination & $(148 / 180)$ & $(96 / 100)$ & $(244 / 280)$ & $(148 / 152)$ & 90.57 \\
& 94.44 & 96.00 & 95.00 & 97.70 & $(96 / 106)$ \\
\hline
\end{tabular}

\section{CCAT2 relative expression in postoperative CC patients} and recurrent/metastatic CC patients

The median value of serum CCAT2 relative expression in 165 postoperative CC patients was 0.790 (0.607,
1.165), showing a significant difference from that in primary CC patients $(P<0.0001)$ (Fig. 5$)$. The median value of serum CCAT2 relative expression in 44 recurrent and metastatic CC patients was 1.752 (1.003, 


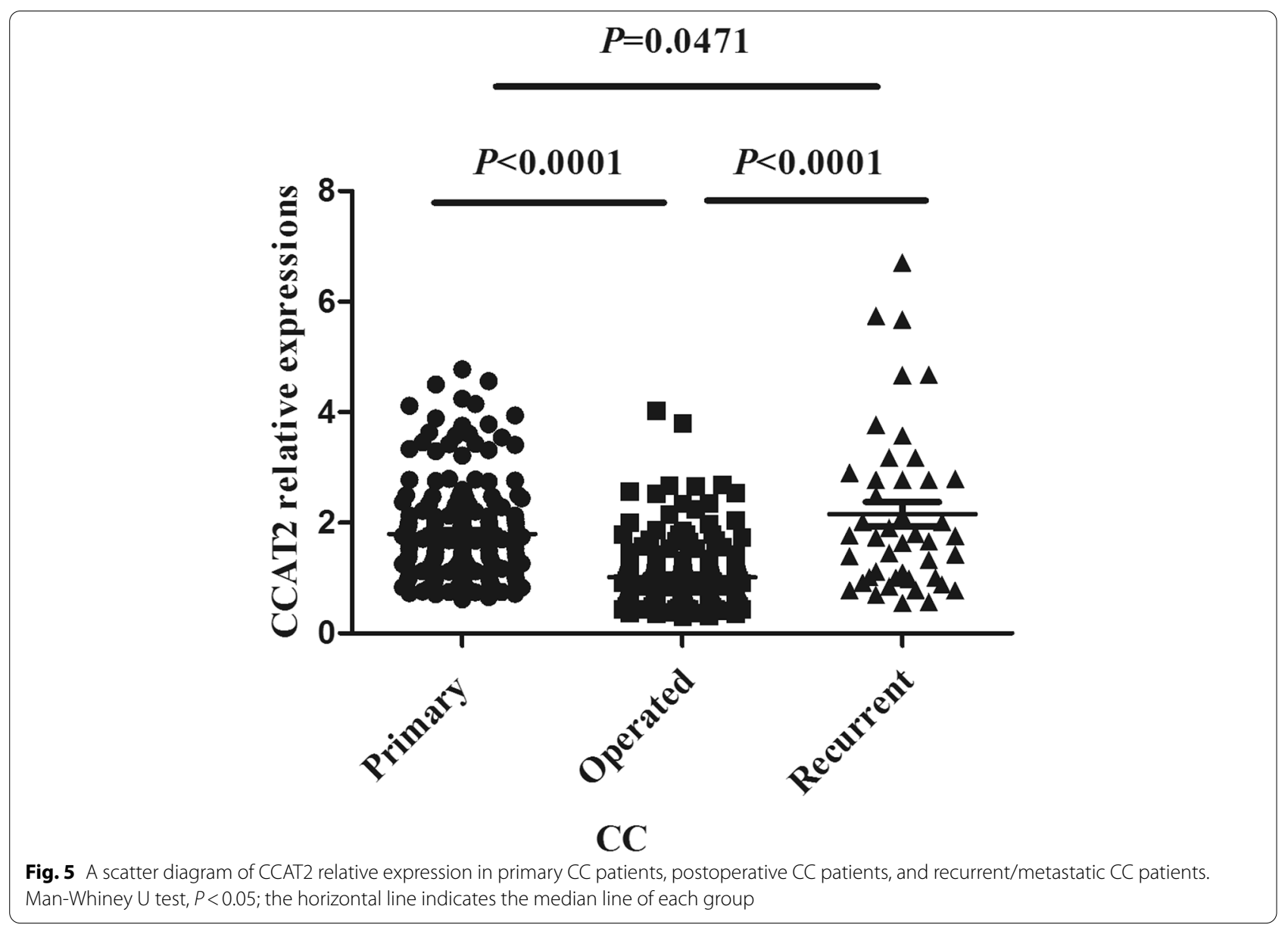

2.787), showing a significant difference from that in primary CC patients and postoperative $C C$ patients $(P=0.0471, P<0.0001)$ (Fig. 5).

\section{CCAT2 dynamic in postoperative CC patients}

Pre- and postoperative CCAT2 was measured in 30 of the 180 primary $\mathrm{CC}$ who received surgery and were followed up. CCAT2 was determined before surgery (day 0 ) in these patients, ranging from 0.732 to 4.5 (median 1.651). At 5-10 days after surgical intervention, a significant decreasing was observed in these subjects. CCAT2 ranged from 0.325 to 2.694 (median 0.786). At 30-60 days after surgery, CCAT2 ranged from 0.444 to 1.779 (median 0.823 ). CCAT2 ranged from 0.442 to 1.079 (median 0.682) during 90-120 days post-operation, and between 0.298 and 1.421 (median 0.585 ) during 150-180 days post-operation (Fig. 6). There was a general trend that CCAT2 was significantly higher before surgery, and decreased progressively in the follow-up period after surgery.

\section{Prognostic value of CCAT2 in CC patients}

We compared the overall survival times between 154 CC patients who expressed high or low expression levels of CCAT2 and SCC based on extensive clinical follow-up data. A Kaplan-Meier survival curve showed that the overall survival rate of $\mathrm{CC}$ patients in high CCAT2 expression group markedly decreased as compared with that of low CCAT2 expression group $(P=0.026)$ (Fig. 7$)$. In addition, univariate analysis and multivariate analyses were per-formed by a Cox proportional hazards regression model to further assess the prognostic value of CCAT2. In the univariate analysis, FIGO stage $(P=0.021)$, lymph node metastasis $(P=0.001)$ and CCAT2 $(P=0.032)$ were associated with OS. In the multivariate analysis, lymph node metastasis $(\mathrm{HR}=0.553,95 \% \mathrm{CI}: 1.684-14.690$, $P=0.004$ ) were independent factors associated with OS, but the influence of CCAT2 $(\mathrm{HR}=0.654,95 \% \mathrm{CI}$ : $0.737-9.575, P=0.135)$ on OS was lost (Table 4$)$. Thus, our results may indicate that CCAT2 was not an independent prognostic factor for $\mathrm{CC}$. 


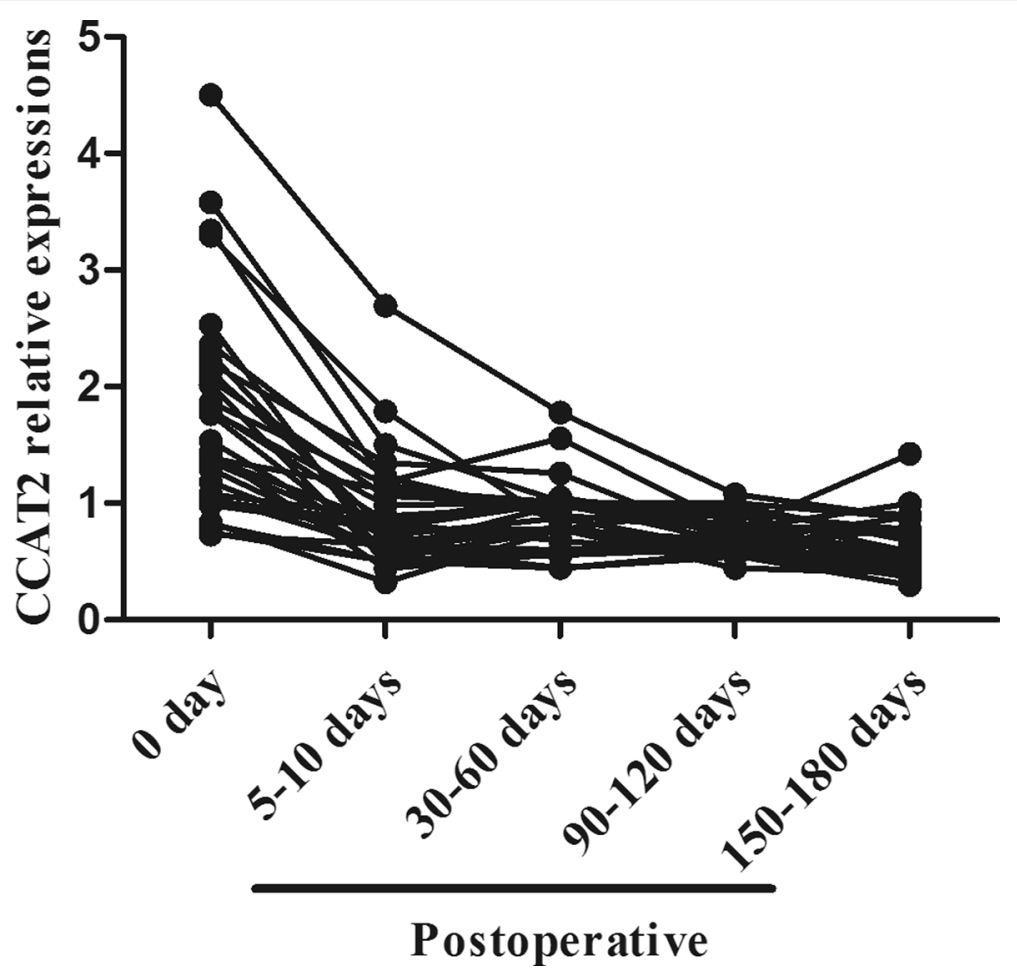

Fig. 6 A straight line diagram of serum CCAT2 relative expression in the 30 follow-up primary CC patients

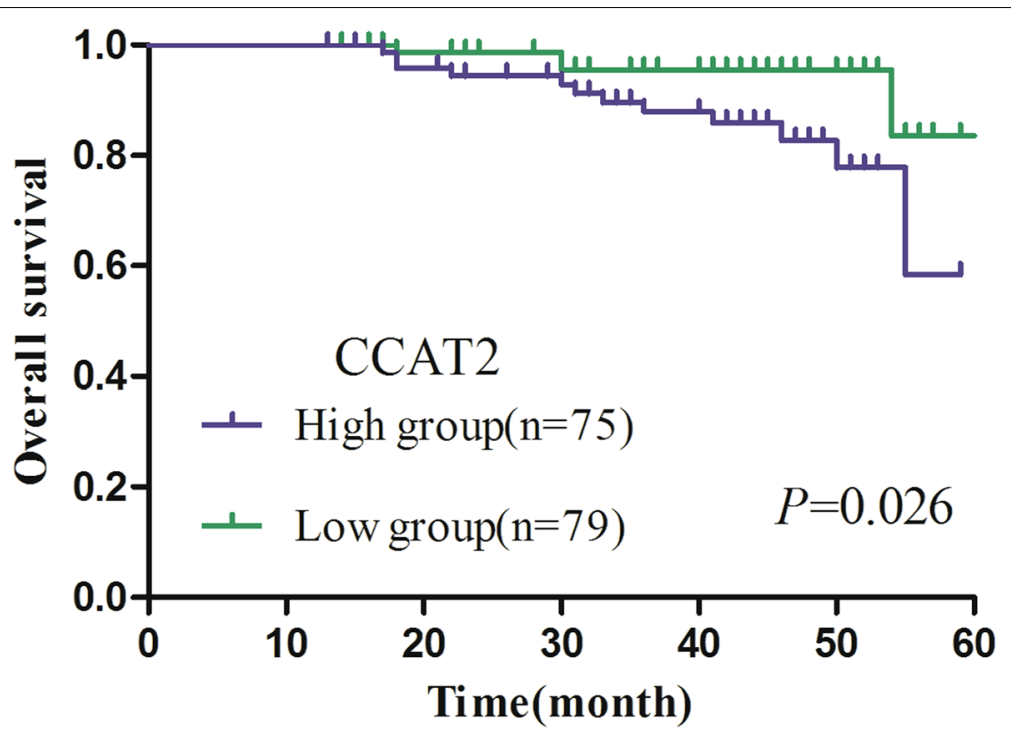

Fig. 7 A Kaplan-Meier curve of CCAT2 for the overall survival of 154 patients with cervical cancer (CC)

\section{Discussion}

Tremendous progress has been made in the research of lncRNAs. Scientists have discovered that lncRNAs are functional transcripts that play key roles in gene regulation and therefore are regarded as new regulators of gene expression. In addition, they are closely related to tumor development and progression [8]. Kim et al. [9] found that HOXA11-AS expression in CC patients was higher than that in the control group. In vitro experiments showed that HOXA11-AS overexpression promoted cell 
Table 4 Univariate multivariate analysis of prognostic factors on OS by the Cox regression model

\begin{tabular}{|c|c|c|c|c|c|c|}
\hline \multirow[t]{2}{*}{ Variables } & \multicolumn{3}{|c|}{ Univariate analysis } & \multicolumn{3}{|c|}{ Multivariate analysis } \\
\hline & HR & $95 \% \mathrm{Cl}$ of $\mathrm{HR}$ & $P$ value & HR & $95 \% \mathrm{Cl}$ of $\mathrm{HR}$ & $P$ value \\
\hline Age & 0.142 & $0.450-3.251$ & 0.706 & & & \\
\hline Tumor size & 0.265 & $0.472-3.620$ & 0.607 & & & \\
\hline FIGO stage & 5.310 & $1.215-11.113$ & $0.021^{*}$ & 0.671 & $0.773-10.727$ & 0.115 \\
\hline pathological types & 0.549 & $0.183-2.151$ & 0.459 & & & \\
\hline Lymph node metastasis & 15.260 & $2.672-19.329$ & $0.001^{*}$ & 0.553 & $1.684-14.690$ & $0.004^{*}$ \\
\hline CCAT2 & 4.606 & $1.114-10.774$ & $0.032^{*}$ & 0.654 & $0.737-9.575$ & 0.135 \\
\hline SCC & 0.042 & $0.407-3.025$ & 0.838 & & & \\
\hline
\end{tabular}

${ }^{*} P<0.05$

proliferation, migration and invasion, while HOXA11-AS knockout inhibited these biological properties. Jin et al [10] found that the expression level of TCONS_00026907 in CC tissue was significantly higher than that in the para-carcinoma tissue, and that patients in the highexpression group had a lower survival rate, while silencing TCONS_00026907 expression and overexpressing miR-143-5p inhibited CC cell proliferation, migration and invasion, and promoted cell apoptosis. Hu et al. [11] reported significantly high expression of taurine-upregulated gene 1 (TUG1) in the CC tissue and that was correlated with tumor size, FIGO stage and LNM. Chen et al. [12] found that high expression of lncRNA cervical carcinoma high-expressed 1 (CCHE1) in CC tissue was correlated with FIGO stage, tumor size, LNM and human papillomavirus. Kaplan-Meier survival curve showed that patients with low CCHE1 levels had better OS and recurrence-free survival (RFS).

CCAT2 is found to play an oncogene role in multiple cancers. Zhao et al. [13] reported that the nucleus and cytoplasmic $\beta$-catenin ptoein level in CCAT2 group was reduced and the Wnt signaling pathway was inhibited significantly, which had a synergistic effect with the Wnt signal inhibitor FH535. CCAT2 promoted the occurrence of non small-cell lung cancer (NSCLC) by regulating the Wnt/ $\beta$-catenin signaling pathway. Wang et al. [14] reported that CCAT2 promoted epithelial-mesenchymal transition (EMT) of gastric cancer cells through downregualting the expression of E-cadherin and upregulating Zinc finger E-box binding homebox 2 (ZEB2), Vimentin and N-cadherin. However, the clinical value of CCAT2 in cancers remains unclear.

The result of RT-qPCR in the present study showed that the relative expression of serum CCAT2 was 1.616 in 180 primary CC patients, 0.795 in 80 CIN patients, and 0.727 in 100 health controls. Mann-Whitney U test showed that the serum CCAT2 relative expression level in primary $\mathrm{CC}$ patients was significantly higher than that in CIN patients and healthy controls $(P<0.001)$. Wang et al. [15] used tumor xenografts and immunohistochemical methods to determine the effect of CCAT2 gene knockout on tumor growth in vivo. The expression of CCAT2 was up-regulated in CC cells and tissues. The cholecystokinin (CCK8) result by Wu et al. [16] showed that CCAT2 knockout inhibited the proliferation of $\mathrm{HeLa}, \mathrm{CaSki}$ and SiHa cells and promoted the proliferation and survival of CC cells. Łaźniak et al. [17] observed that the G variant of CCAT2 rs6983267 SNP induced cervical squamous cell carcinoma cells to diffuse to the surrounding tissues and promoted the rapid growth of low-grade tumor cells. All these findings provide theoretical clues to support the possibility of CCAT2 as an adjuvant diagnostic marker for CC. In addition, we found that CCAT2 relative expression was positively correlated with tumor FIGO stage, SCC-Ag and LNM (all $P<0.05$ ), suggesting that CCAT2 expression level may prove to be an adjuvant marker for monitoring the degree of malignancy and disease progression.

CCAT2 relative expression was correlated with the SCC content in primary CC patients $(P<0.0001$, $\left.r^{2}=0.120\right)$ and not with the CA125 content $(P=0.926$, $\left.r^{2}<0.0001\right)$. The AUC value of CCAT2 in differential diagnosis between primary $\mathrm{CC}$ patients and healthy controls was 0.897 (95\%CI:0.862-0.933) and the cutoff value was 1.102; under this critical value, the sensitivity, specificity, accuracy, positive prediction and negative prediction were $73.33,87.00,78.21,91.03$ and $64.44 \%$ respectively, indicating that CCAT2 has a relatively high sensitivity and specificity. Next a combined analysis of CCAT2, CA125 and SCC was performed using a tandem model of the three markers. The sensitivity, specificity, accuracy, positive prediction and negative prediction of this panel in differentiating the healthy controls were 94.44, 96.00, 95.00, 97.70 and $90.57 \%$. More importantly, combination detection of CCAT2, CA125 and SCC could greatly improve the diagnostic efficiency of primary CC. 
The median value of serum CCAT2 relative expression in 165 postoperative CC patients was 0.790 , showing a significant decreasing as compared with that in the preoperative CC patients. The median of CCAT2 relative expression in the 44 recurrent and metastatic CC patients was 1.752 , showing significant increasing from that in the primary CC patients and postoperative $\mathrm{CC}$ patients, suggesting that serum CCAT2 has certain significance in assessing the prognosis of CC patients.

Finally, we assessed the postoperative dynamic change of CCAT2 in 30 of the 180 primary CC patients through 6-month follow-up observation. The results showed that there was a significantly decreasing tendency in CCAT2 expression during D5-10 post-operation, which may be because the serum content of CCAT2 was decreased after resection of the tumor. Serum CCAT2 showed a slowly decreasing tendency during D30-180 post-operation. In all but one patient, imaging and clinical analysis suggested the possibility of cancer recurrence. Therefore, detection of dynamic change in serum CCAT2 could be used to dynamically monitoring the postoperative prognosis of surgical treatment in CC patients, though larger-sample studies are required to verify our conclusion. In the prognostic aspect, Kaplan-Meier survival curve showed that the overall survival rate of CC patients in high CCAT2 expression group markedly decreased as compared with that of low CCAT2 expression group. Univariable analysis showed that FIGO stage, Lymph node metastasis and CCAT2 were all significantly associated with OS.

CCAT2 was also found to be highly expressed in pancreatic ductal adenocarcinoma, ovarian cancer tissues and bladder cancer [18-20]. Above all, serum CCAT2 relative expression is to some extent associated with the development and progression of $\mathrm{CC}$, suggesting that CCAT2 may prove to be an important biomarker. Analysis and assessment of the CCAT2 value in treatment and prognostic prediction of CC showed that CCAT2 detection in combination with CA125 and SCC could improve the diagnostic efficiency of CC. Our study also lays a foundation for further clarification of the action mechanism of CCAT2 in CC. It is well known that human papillomavirus (HPV) has been identified as the main factor leading to cervical cancer. Many patients with cervical cancer are positive for high-risk HPV. Qu et al. [21] found that lncRNA small nucleolar RNA host gene 8 (SNHG8) recruited enhancer of zeste homolog 2 (EZH2) to downregulate reversion-inducing cysteine-rich protein with Kazal motifs (RECK) expression, leading to HPV-induced $\mathrm{CC}$ aggravation. This will be a good idea for the future study of the mechanism between CCAT2 and HPV in cervical cancer.
Nevertheless, the above results have some limitations. First, all samples were from the same hospital and further prospective multicenter studies will be needed. In addition, our assessment was based on the relative expression of CCAT2 in serum, and its expression in primary CC tumor tissues and cells remains to be further studies for the sake of providing more solid evidence for early diagnosis and prognostic prediction of CC.

\section{Acknowledgements \\ We would like to thank Affiliated Tumor Hospital of Nantong University and Affiliated Hospital of Nantong University.}

\section{Authors' contributions}

$X L C$ and JYZ researched the literature and conceived the study. XLC, JY, MQJ,XJS, JYZ and SQJ were involved in protocol development, gaining ethical approval, patient recruitment and data analysis. XLC wrote the first draft of the manuscript. All authors reviewed and edited the manuscript and approved the final version of the manuscript.

\section{Funding}

This project was supported by the National Natural Science Foundation of China (No. 82072363); the Nantong Science and Technology Project (MS12021002); the mandatory project of Nantong Municipal Health and Family Planning Commission (WKZL2018051).

\section{Availability of data and materials}

Data sharing is not applicable to this article as no datasets were generated or analysed during the current study.

\section{Declarations}

\section{Ethics approval and consent to participate}

The study was approved by the Ethics Committee of Affiliated Tumor Hospital of Nantong University.

\section{Consent for publication}

Not applicable.

\section{Competing interests}

The authors declare that they have no competing interests.

\section{Author details}

${ }^{1}$ Department of Laboratory Medicine, Affiliated Tumor Hospital of Nantong University, \#48 West Qingnian Road, Nantong 226019, Jiangsu Province, China. ${ }^{2}$ Gynecology of Affiliated Tumor Hospital of Nantong University, \#48 West Qingnian Road, Nantong 226019, Jiangsu Province, China. ${ }^{3}$ Laboratory Medicine Center, Affiliated Hospital of Nantong University, \#20 Xisi Road, Nantong 226001, Jiangsu Province, China.

Received: 24 November 2020 Accepted: 16 January 2022

Published online: 03 February 2022

\section{References}

1. Torre LA, Bray F, Siegel RL, Ferlay J, Lortet-Tieulent J, Jemal A. Global cancer statistics, 2012. CA Cancer J Clin. 2015;65(2):87-108.

2. He P, Xiong GG, Guo W, Jiang GJ, Li Y, Li H. Long non-coding RNA CCAT2 promotes prostate cancer cell proliferation and invasion by regulating the Wnt/ $\beta$-catenin signaling pathway. Oncol Lett. 2020;20(4):97.

3. Sun SL, Shu YG, Tao MY. LncRNA CCAT2 promotes angiogenesis in glioma through activation of VEGFA signalling by sponging miR-424. Mol Cell Biochem. 2020;468(1-2):69-82.

4. Xu Z, Liu C, Zhao Q, Lü JH, Ding X, Luo A, et al. Long non-coding RNA CCAT2 promotes oncogenesis in triple-negative breast cancer by regulating stemness of cancer cells. Pharmacol Res. 2020;152:104628. 
5. Roxana SR, Anieta MS, Maxime PL, Oana T, Cristina I, Riccardo S, et al. CCAT2, a novel long non-coding RNA in breast cancer: expression study and clinical correlations. Oncotarget. 2013;4:1748-62.

6. Fu CB, Xu X, Lu WJ, Nie L, Yin T, Wu DD. Increased expression of long noncoding RNA CCAT2 predicts poorer prognosis in patients with hepatocellular carcinoma. Medicine (Baltimore). 2019;98(42):17412.

7. Edge SB, Compton CC. AJCC cancer staging manual The American Joint Committee on Cancer: the 7th Edition of the AJCC Cancer Staging Manual and the Future of TNM. Ann Surg Oncol. 2010;17(6):1471-4.

8. Wang KC, Chang HY. Molecular mechanisms of long non-coding RNAs. Mol Cell. 2011;43(6):904-14.

9. Kim HJ, Eoh KJ, Kim LK, Nam EJ, Yoon SO, Kim KH, et al. The long noncoding RNA HOXA11 antisense induces tumor progression and stemness maintenance in cervical cancer. Oncotarget. 2016;7(50):83001-16.

10. Jin XJ, Chen $X G$, Hu Y, Ying FR, Zou R, Lin F, et al. LncRNATCONS_00026907 is involved in the progression and prognosis of cervical cancer through inhibiting miR-143-5p. Cancer Med. 2017;6(6):1409-23.

11. Hu YY, Sun XW, Mao CC, Guo GQ, Ye SS, Xu JF, et al. Upregulation of long noncoding RNA TUG1 promotes cervical cancer cell proliferation and migration. Cancer Med. 2017;6(2):471-82.

12. Chen $Y$, Wang $C X$, Sun $X X$, Wang $C$, Liu TF, Wang DJ. Long non-coding RNA CCHE1 overexpression predicts a poor prognosis for cervical cancer. Eur Rev Med Pharmacol Sci. 2017;21(3):479-83.

13. Zhao CL, Qiao CC, Zong LG, Chen YQ. Long non-coding RNA-CCAT2 promotes the occurrence of non-small cell lung cancer by regulating the Wnt/ß-catenin signaling pathway. Oncol Lett. 2018;16:4600-6.

14. Wang YJ, Liu JZ, LV P, Dang Y, Gao JY, Wang Y. Long non-coding RNA CCAT2 promotes gastric cancer proliferation and invasion by regulating the E-cadherin and LATS2. Am J Cancer Res. 2016;6(11):2651-60.

15. Wang J, Liu Y, Cai HB, Jiang H, Li W, Shi YY. Long coding RNA CCAT2 enhances the proliferation and epithelial-mesenchymal transition of cervical carcinoma cells via the microRNA-493-5p/CREB1 axis. Bioengineered. 2021;12(1):6264-74.

16. Wu L, Jin LX, Zhang WM, Zhang LF. Roles of long non-coding RNA CCAT2 in cervical Cancer cell growth and apoptosis. Med Sci Monit. 2016;22:875-9.

17. Łaźniak S, Lutkowska A, Wareńczak-Florczak Z, Sowińska A, Tsibulski A, Roszak A. Etal. The association of CCAT2 rs6983267 SNP with MYC expression and progression of uterine cervical cancer in the polish population. Arch Gynecol Obstet. 2018;297:1285-92.

18. Cai Y, Li XM, Shen P, Zhang D. CCAT2 is an oncogenic long non-coding RNA in pancreatic ductal adenocarcinoma. Biol Res. 2018;51(1):1.

19. Wang L, Zhou S, Guo B. Vitamin D suppresses ovarian Cancer growth and invasion by targeting long non-coding RNA CCAT2. Int J Mol Sci. 2020;21(7):2334

20. Li JF, Zhuang CL, Liu YC, Chen MW, Zhou Q, Chen ZC, et al. shRNA targeting long non-coding RNA CCAT2 controlled by tetracyclineinducible system inhibits progression of bladder cancer cells. Oncotarget 2016;7(20):28989-97.

21. Qu XH, Li YY, Wang L, Yuan NG, Ma M, Chen Y. LncRNA SNHG8 accelerates proliferation and inhibits apoptosis in HPV-induced cervical cancer through recruiting EZH2 to epigenetically silence RECK expression. J Cell Biochem. 2020;121(10):4120-9.

\section{Publisher's Note}

Springer Nature remains neutral with regard to jurisdictional claims in published maps and institutional affiliations.

Ready to submit your research? Choose BMC and benefit from:

- fast, convenient online submission

- thorough peer review by experienced researchers in your field

- rapid publication on acceptance

- support for research data, including large and complex data types

- gold Open Access which fosters wider collaboration and increased citations

- maximum visibility for your research: over $100 \mathrm{M}$ website views per year

At $\mathrm{BMC}$, research is always in progress.

Learn more biomedcentral.com/submissions 